\title{
Evolution speed of open quantum dynamics
}

\author{
Dorje C. Brody $\oplus^{1}$ and Bradley Longstaff $\odot^{2}$ \\ ${ }^{1}$ Department of Mathematics, University of Surrey, Guildford GU2 7XH, United Kingdom \\ ${ }^{2}$ Department of Mathematics, Imperial College London, London SW7 2AZ, United Kingdom
}

(Received 14 June 2019; published 25 November 2019)

\begin{abstract}
The space of density matrices is embedded in a Euclidean space to deduce the dynamical equation satisfied by the state of an open quantum system. The Euclidean norm is used to obtain an explicit expression for the speed of the evolution of the state. The unitary contribution to the evolution speed is given by the modified skew information of the Hamiltonian, while the radial component of the evolution speed, connected to the rate at which the purity of the state changes, is shown to be determined by the modified skew information of the Lindblad operators. An open-system analog of the quantum navigation problem is posed, and a perturbative analysis is presented to identify the amount of change on the speed. Properties of the evolution speed are examined further through example systems, showing that the evolution speed need not be a decreasing function of time.
\end{abstract}

DOI: 10.1103/PhysRevResearch.1.033127

\section{INTRODUCTION}

Understanding the speed of the evolution of a quantum state is of interest for a variety of reasons in quantum information science. As well as being of interest in its own right [1,2], in implementing quantum algorithms for establishing communication and performing computation, for instance, the evolution speed determines how fast a given task can be processed. The speed also determines the sensitivity of quantum states against time evolution, and this information can be used to determine error bounds on quantum state estimation [3].

In the case of a pure state undergoing a unitary time evolution, the evolution speed of the state in Hilbert space was identified by Anandan and Aharonov as twice the energy uncertainty of the system [4]. In the case of a mixed state, the speed of unitary time evolution in Hilbert space is somewhat reduced to twice the Wigner-Yanase skew information [5,6]. Interests in the evolution speed in the context of quantumstate estimation grew rapidly after the work in Ref. [7] that connected the estimation problem to the geometry of the quantum state space. By now there is a substantial body of literature that clarifies various aspects of the speed of unitary time evolution (see, e.g., Ref. [8] and references cited therein).

More recently, inspired in part by the desire to understand fundamental quantum limits to implementing quantum processes in more realistic environments, research activity into the study of evolution speed of open quantum systems has intensified (see Ref. [9] and references cited therein). In this connection, it is worth noting that the notion of speed, which is the ratio of distance and time, crucially depends on the

Published by the American Physical Society under the terms of the Creative Commons Attribution 4.0 International license. Further distribution of this work must maintain attribution to the author(s) and the published article's title, journal citation, and DOI. choice of the metric on the space of quantum states. For pure states, there is little ambiguity to the matter on account of the existence of a unique unitary-invariant Fubini-Study metric on the space of pure states [10]. However, for mixed-state density matrices, the structure of the state space is more intricate, and there is a range of different metrics one can impose, whose merits are dependant on the particular application one might consider. For instance, in Ref. [11] the authors consider the parameter sensitivity of the state by examining the Fisher information associated with an open system dynamics. The trace norm of the difference of two density matrices is considered in Ref. [12] to define distance, which is used to bound the evolution speed of an open-system dynamics. In Ref. [13] the purity of the state is used to define distance, and an upper bound for the speed of evolution is obtained. In Ref. [14] Uhlmann's fidelity [15,16] between the initial state, assumed pure, and the terminal state is taken to define distance to obtain a bound on the evolution speed for general Markovian open dynamics. The fidelity-based measure is also considered in Ref. [17] where the minimum evolution time for general open-system dynamics is investigated, showing that the closely-related work of Ref. [18] does not reproduce their results. This is natural because in Ref. [18] the relative purity is used to define distance, and hence one does not a priori expect the results to coincide. In Ref. [19] the evolution speed of the state under the influence of non-Hermitian Hamiltonian is worked out.

In the present paper, we regard the density matrix as representing a vector in a Euclidean space, and take the natural Euclidean metric as defining the distance measure. In this setup, we consider a one-parameter family of states generated by a general open-system dynamics, and work out the explicit expression for the evolution speed, which consists of three terms: one associated with the unitary time evolution, given by the modified skew information of the Hamiltonian; one associated with the environmental influences generated by Lindblad operators; and one associated with the competition between the two. We also work out the radial component of 
the speed, which is shown to be related to the speed of the change of the purity $\operatorname{tr}\left(\hat{\rho}^{2}\right)$ of the state. Surprisingly, the rate of purity change is given by the modified skew information of the Lindblad operators. We then calculate the changes to the speed induced by a small perturbation of the Hamiltonian, when the ambient environmental influences, characterized by the Lindblad operators, cannot be controlled (an analog of the Zermelo navigation problem [20] for open quantum systems). Quantum control under an open environment is a subject of much recent activity (see, e.g., Refs. [9,21]), and the navigation problem introduced here offers a new class of problems to explore. The behaviors of the evolution speed are then studied in example systems. For a PT-symmetric quantum system, we show that the existence of a phase transition leads to qualitatively different behaviors of the speed; while in a Bose-Hubbard system coupled to a reservoir we show that the evolution speed need not be decreasing in time, contrary to what other studies have suggested.

\section{DYNAMICS OF THE STATE}

We begin by remarking that the space of density matrices in a Hilbert space $\mathcal{H}^{n}$ of dimension $n$ forms a subset of the interior of a sphere $S^{n^{2}-2}$ in a Euclidean space $\mathbb{R}^{n^{2}-1}$ [10]. Thus every density matrix $\hat{\rho}$ can be thought of as being represented by a vector $\boldsymbol{r} \in \mathbb{R}^{n^{2}-1}$. There are various ways in which we can choose a system of coordinates for $\mathbb{R}^{n^{2}-1}$, but here we consider the generalisation of the Bloch vector representation [22]. For this purpose, we let $\left\{\hat{\sigma}_{j}\right\}_{j=0, \ldots, n^{2}-1}$ be an orthonormal basis for the linear space of bounded operators on $\mathcal{H}^{n}$ equipped with the Hilbert-Schmidt inner product $\langle\hat{\sigma}, \hat{\tau}\rangle=\operatorname{tr}\left(\hat{\sigma}^{\dagger} \hat{\tau}\right)$. We set $\hat{\sigma}_{0}=n^{-1 / 2} \mathbb{1}$, hence the operators $\left\{\hat{\sigma}_{j}\right\}_{j=1, \ldots, n^{2}-1}$ are trace free, and together they satisfy the orthonormality condition $\left\langle\hat{\sigma}_{i}, \hat{\sigma}_{j}\right\rangle=\delta_{i j}$. For $n=2$, we may set $\left\{\hat{\sigma}_{j}\right\}_{j=1, \ldots, 3}$ to $1 / \sqrt{2}$ times the Pauli matrices; for $n=3$ we may set $\left\{\hat{\sigma}_{j}\right\}_{j=1, \ldots, 8}$ to $1 / \sqrt{2}$ times the Gell-Mann matrices, and so on [23]. An arbitrary density matrix $\hat{\rho}$ can then be expressed in the form

$$
\hat{\rho}=\frac{1}{\sqrt{n}} \hat{\sigma}_{0}+\sum_{j=1}^{n^{2}-1} r_{j} \hat{\sigma}_{j}
$$

where $r_{j}=\operatorname{tr}\left(\hat{\rho} \hat{\sigma}_{j}\right), j=1, \ldots, n^{2}-1$, are the components of the vector $\boldsymbol{r} \in \mathbb{R}^{n^{2}-1}$. For a pure state, we have

$$
1=\operatorname{tr}\left(\hat{\rho}^{2}\right)=\frac{1}{n}+\sum_{j=1}^{n^{2}-1} r_{j}^{2}
$$

from which it follows that the squared radius of the sphere $S^{n^{2}-2}$ in $\mathbb{R}^{n^{2}-1}$ is given by $1-n^{-1}$.

We now consider a one-parameter family of density matrices $\hat{\rho}(t)$ parameterized by time $t$ that satisfies the dynamical equation

$$
\partial_{t} \hat{\rho}=-i[\hat{H}, \hat{\rho}]+\sum_{k}\left[\hat{L}_{k} \hat{\rho} \hat{L}_{k}^{\dagger}-\frac{1}{2}\left(\hat{L}_{k}^{\dagger} \hat{L}_{k} \hat{\rho}+\hat{\rho} \hat{L}_{k}^{\dagger} \hat{L}_{k}\right)\right]
$$

along with an initial condition $\hat{\rho}(0)$. The unitary part of the dynamics is described by the Hamiltonian $\hat{H}$, and $\left\{\hat{L}_{k}\right\}$ is a family of Lindblad operators characterising the system interaction with its environment. Our first objective is to identify the linear differential equation satisfied by the state $\boldsymbol{r}$ that corresponds to the evolution equation (3) for the density matrix. For this purpose, we substitute (1) in (3) to obtain

$$
\begin{aligned}
\sum_{j=1}^{n^{2}-1} \dot{r}_{j} \hat{\sigma}_{j}= & -i \sum_{j=1}^{n^{2}-1}\left[\hat{H}, \hat{\sigma}_{j}\right] r_{j}+\frac{1}{n} \sum_{k}\left[\hat{L}_{k}, \hat{L}_{k}^{\dagger}\right] \\
& +\sum_{j=1}^{n^{2}-1} \sum_{k}\left[\hat{L}_{k} \hat{\sigma}_{j} \hat{L}_{k}^{\dagger}-\frac{1}{2}\left(\hat{L}_{k}^{\dagger} \hat{L}_{k} \hat{\sigma}_{j}+\hat{\sigma}_{j} \hat{L}_{k}^{\dagger} \hat{L}_{k}\right)\right] r_{j} .
\end{aligned}
$$

We multiply $\hat{\sigma}_{i}$ to both sides of (4) and take the trace, using the orthonormality relation $\left\langle\hat{\sigma}_{i}, \hat{\sigma}_{j}\right\rangle=\delta_{i j}$, to deduce that $r_{i}$ satisfies the differential equation

$$
\dot{r}_{i}=\sum_{j=1}^{n^{2}-1} \Lambda_{i j} r_{j}+b_{i}
$$

where

$$
\begin{aligned}
\Lambda_{i j}= & \operatorname{tr}\left[-i\left[\hat{\sigma}_{j}, \hat{\sigma}_{i}\right] \hat{H}+\sum_{k} \hat{L}_{k} \hat{\sigma}_{j} \hat{L}_{k}^{\dagger} \hat{\sigma}_{i}\right. \\
& \left.-\frac{1}{2} \sum_{k}\left(\hat{L}_{k}^{\dagger} \hat{L}_{k} \hat{\sigma}_{j} \hat{\sigma}_{i}+\hat{L}_{k}^{\dagger} \hat{L}_{k} \hat{\sigma}_{i} \hat{\sigma}_{j}\right)\right]
\end{aligned}
$$

is a real matrix and

$$
b_{i}=\frac{1}{n} \sum_{k} \operatorname{tr}\left(\left[\hat{L}_{k}, \hat{L}_{k}^{\dagger}\right] \hat{\sigma}_{i}\right)
$$

is a real vector. If the Lindblad operators are Hermitian, or more generally if they are normal, then we have $b_{j}=0$. There are also other circumstances in which $\boldsymbol{b}$ vanishes, for instance when there are two Lindblad operators given by $\hat{L}_{1}=\hat{\sigma}_{+}$and $\hat{L}_{2}=\hat{\sigma}_{-}$, where $\hat{\sigma}_{ \pm}=\hat{\sigma}_{x} \pm i \hat{\sigma}_{y}$.

From the linearity of the dynamics we can think of the right side of (3) as representing the action of a Liouville operator $\mathcal{L}$ (cf. Ref. [24]) on $\hat{\rho}$, and write $\partial_{t} \hat{\rho}=\mathcal{L} \hat{\rho}$ for (3), where $\hat{\rho}$ is viewed as a vector on which the linear operator $\mathcal{L}$ acts. The components $\mathcal{L}_{i j}$ of $\mathcal{L}$ in the basis $\left\{\hat{\sigma}_{j}\right\}$ are given by $\mathcal{L}_{i j}=\left\langle\hat{\sigma}_{i}, \mathcal{L} \hat{\sigma}_{j}\right\rangle=\operatorname{tr}\left(\hat{\sigma}_{i} \mathcal{L} \hat{\sigma}_{j}\right)$, and we have $(\mathcal{L} \hat{\rho})_{j}=\sum_{i} \operatorname{tr}\left(\hat{\sigma}_{j} \mathcal{L} \hat{\sigma}_{i}\right) \operatorname{tr}\left(\hat{\sigma}_{i} \hat{\rho}\right)$. It follows that $(\mathcal{L} \hat{\rho})_{0}=0$, because $\operatorname{tr}(\mathcal{L} \hat{\xi})=0$ for any $\hat{\xi}$. Therefore, writing

$$
\dot{r}_{j}=\sum_{i=1}^{n^{2}-1} \mathcal{L}_{j i} r_{i}+\frac{1}{\sqrt{n}} \mathcal{L}_{j 0}
$$

we deduce from (5) that the matrix elements of the Liouville operator are given by $\mathcal{L}_{j i}=\Lambda_{j i}$ for $i, j \neq 0$ and $\mathcal{L}_{j 0}=\sqrt{n} b_{j}$. Because the real matrix $\mathcal{L}_{j i}$ is not symmetric, its eigenvalues are either real or else come in complex conjugate pairs, such that the real parts of the eigenvalues are nonpositive, thus generating a completely positive map on the space of density matrices [25]. 


\section{EVOLUTION SPEED}

Having obtained the dynamical equation satisfied by the state vector $\boldsymbol{r} \in \mathbb{R}^{n^{2}-1}$ we are now in the position to determine the squared speed of evolution:

$$
v^{2}(t)=\sum_{j=1}^{n^{2}-1} \dot{r}_{j}^{2}=\operatorname{tr}\left[(\mathcal{L} \hat{\rho})^{2}\right] .
$$

To proceed let us write the time derivative of the state $\hat{\rho}$ in the form $\mathcal{L} \hat{\rho}=-i[\hat{H}, \hat{\rho}]+\mathcal{D} \hat{\rho}$, thus isolating the dissipator term

$$
\mathcal{D} \hat{\rho}=\sum_{k}\left[\hat{L}_{k} \hat{\rho} \hat{L}_{k}^{\dagger}-\frac{1}{2}\left(\hat{L}_{k}^{\dagger} \hat{L}_{k} \hat{\rho}+\hat{\rho} \hat{L}_{k}^{\dagger} \hat{L}_{k}\right)\right]
$$

from the unitary part of $\mathcal{L}$. Squaring $\mathcal{L} \hat{\rho}$ and taking the trace, we obtain

$$
\begin{aligned}
v^{2}(t)= & 2\left[\operatorname{tr}\left(\hat{H}^{2} \hat{\rho}^{2}\right)-\operatorname{tr}(\hat{H} \hat{\rho} \hat{H} \hat{\rho})\right] \\
& -2 i \operatorname{tr}(\hat{\rho}[\mathcal{D} \hat{\rho}, \hat{H}])+\operatorname{tr}\left[(\mathcal{D} \hat{\rho})^{2}\right] .
\end{aligned}
$$

There are three terms contributing to the speed of evolution; the first arising purely from the unitary evolution and the third arising purely from the dissipator term, while the second term represents in some sense the competition between the Hamiltonian and the Lindblad operators. To see this we note that the cross term in Eq. (11) can alternatively be written in the form

$$
-2 i \sum_{k}\left[\operatorname{tr}\left(\hat{\rho}\left[\hat{L}_{k} \hat{\rho} \hat{L}_{k}^{\dagger}, \hat{H}\right]\right)+\frac{1}{2} \operatorname{tr}\left(\hat{\rho}^{2}\left[\hat{H}, \hat{L}_{k}^{\dagger} \hat{L}_{k}\right]\right)\right],
$$

which vanishes if $\hat{L}_{k}^{\dagger}=\hat{L}_{k}$ and $\left[\hat{L}_{k}, \hat{H}\right]=0$, thus representing the lack of compatibility between $\hat{H}$ and $\left\{\hat{L}_{k}\right\}$.

The contribution to the squared speed from the unitary evolution (the first term in Eq. (11)) has recently been identified in Ref. [26] as the speed of the evolution of the state, when the distance is measured with respect to the Euclidean angular separation. This term resembles, but is different from, the Wigner-Yanase skew information $I=\operatorname{tr}\left(\hat{H}^{2} \hat{\rho}\right)-$ $\operatorname{tr}(\hat{H} \sqrt{\hat{\rho}} \hat{H} \sqrt{\hat{\rho}})$. The apparent discrepancy between this result and that obtained in Ref. [5] is that here we measure the speed with respect to the Euclidean norm in $\mathbb{R}^{n^{2}-1}$, whereas in Ref. [5] the evolution speed of the state is obtained using the Hilbert space norm. While the latter is more useful in the context of state estimation (because the Fisher-Rao metric for unitary evolution is given by the skew information), as remarked in Ref. [26] for the analysis of the evolution speed and time, the use of the Euclidean metric is computationally more effective for it does not involve taking the square root of the density matrix. We shall refer to $S(X)=\operatorname{tr}\left(\hat{X}^{\dagger} \hat{X} \hat{\rho}^{2}\right)-$ $\operatorname{tr}\left(\hat{X} \hat{\rho} \hat{X}^{\dagger} \hat{\rho}\right)$ as the "modified skew information" for $\hat{X}$, which reduces to the variance $\Delta X^{2}=\left\langle\hat{X}^{\dagger} \hat{X}\right\rangle-\left\langle\hat{X}^{\dagger}\right\rangle\langle\hat{X}\rangle$ for a pure state.

In contrast to unitary time evolution, in an open system the velocity will in general obtain a radial component so that the purity $\operatorname{tr}\left(\hat{\rho}^{2}\right)$ changes. To see this, consider the squared magnitude of the radial velocity $v_{R}^{2}(t)=(\boldsymbol{r} \cdot \dot{\boldsymbol{r}})^{2} /(\boldsymbol{r}$. $\boldsymbol{r})=[\operatorname{tr}(\hat{\rho} \mathcal{L} \hat{\rho})]^{2} / \operatorname{tr}\left[\left(\hat{\rho}-n^{-1} \mathbb{1}\right)^{2}\right]$, which vanishes for unitary dynamics. In Ref. [13] an upper bound for the numerator term $[\operatorname{tr}(\hat{\rho} \mathcal{L} \hat{\rho})]^{2}$ is obtained using Cauchy-Schwarz inequality, but in fact a short calculation shows remarkably that

$$
v_{R}(t)=\sum_{k} \frac{S\left(L_{k}\right)}{\sqrt{\operatorname{tr}\left[\left(\hat{\rho}-n^{-1} \mathbb{1}\right)^{2}\right]}} .
$$

In other words, the speed of the change of the purity is given by twice the modified skew information associated with the Lindblad operators. This is surprising, for, while the unitary flow is linear in $\hat{H}$, the dissipation flow is bilinear in $\hat{L}$ and $\hat{L}^{\dagger}$.

Let us turn to consider optimisation. Two scenarios that might arise in the context of open quantum systems are (i) to maximize the speed over all Liouville operators and (ii) to maximize the speed over all Hamiltonians for a fixed open environment $\left\{\hat{L}_{k}\right\}$. The latter problem arises when an experimentalist has no control over the environmental influences, but nonetheless can set the Hamiltonian so as to implement a rapid state transportation (an open-system analog of the quantum navigation problem [20]). Under a unitary evolution, the solution to problem (i) is obtained by maximising the modified skew information. For an open system, finding general solutions to these problems is nontrivial, in part because of the competition between $\hat{H}$ and $\left\{\hat{L}_{k}\right\}$, i.e., the second term in Eq. (11) can a priori be positive or negative. Nevertheless, progress can be made if we note that the right side of (11) is in fact just the squared length of the sum vector of the Hamiltonian flow $-i[\hat{H}, \hat{\rho}]$ and the Lindblad flow $\mathcal{D} \hat{\rho}$, which implies that subject to finite energy constraints the speed is maximized by minimising the angular separation of these two vectors. Alternatively, we can explore a perturbative analysis. Specifically, for problem (ii), we perturb the Hamiltonian $\hat{H}$ in the direction of $\hat{\Delta}$ by a small amount $\epsilon$, i.e., we let $\hat{H} \rightarrow \hat{H}+\epsilon \hat{\Delta}$, and work out how much the speed changes in the limit $\epsilon \rightarrow 0$. A calculation shows that this is given by

$$
\begin{aligned}
\delta v^{2}= & 2\left[\operatorname{tr}\left((\hat{H} \hat{\Delta}+\hat{\Delta} \hat{H}) \hat{\rho}^{2}\right)-2 \operatorname{tr}(\hat{H} \hat{\rho} \hat{\Delta} \hat{\rho})\right] \\
& -2 i \sum_{k}\left[\operatorname{tr}\left(\hat{\rho}\left[\hat{L}_{k} \hat{\rho} \hat{L}_{k}^{\dagger}, \hat{\Delta}\right]\right)+\frac{1}{2} \operatorname{tr}\left(\hat{\rho}^{2}\left[\hat{\Delta}, \hat{L}_{k}^{\dagger} \hat{L}_{k}\right]\right)\right],
\end{aligned}
$$

which can be used, e.g., to numerically explore the optimal way to modify the Hamiltonian under uncontrollable environmental influences.

\section{EXAMPLES}

We now examine the behavior of the evolution speed via illustrative examples. For the first example we take the Hamiltonian to be $\hat{H}=\frac{1}{2} g \hat{\sigma}_{z}$ and the Lindblad operator to be $\hat{L}=\sqrt{\gamma} \hat{\sigma}_{z}$, thus describing pure dephasing of the two-level system with a decay rate $\gamma$. In this example, we find that $v^{2}(t)=e^{-4 \gamma t}\left(4 \gamma^{2}+g^{2}\right)\left[r_{x}^{2}(0)+r_{y}^{2}(0)\right]$, and hence that $v(t)$ decreases exponentially in time.

As a variant of the previous example, suppose that the Hamiltonian is $\hat{H}=\frac{1}{2} g \hat{\sigma}_{x}$ so that it no longer commutes with the Lindblad operator $\hat{L}=\sqrt{\gamma} \hat{\sigma}_{z}$. This is perhaps the simplest example of a PT-symmetric quantum system admitting a phase transition. In this case, we have

$$
\Lambda=\left(\begin{array}{ccc}
-2 \gamma & 0 & 0 \\
0 & -2 \gamma & -g \\
0 & g & 0
\end{array}\right),
$$




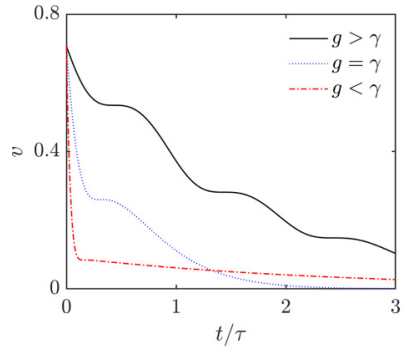

(a) Evolution speed $v$

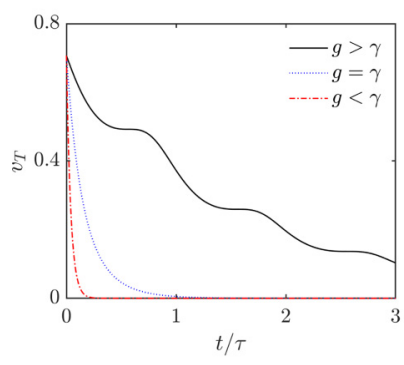

(c) Tangential component $v_{T}$

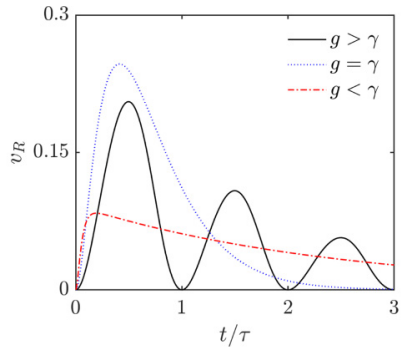

(b) Radial component $v_{R}$

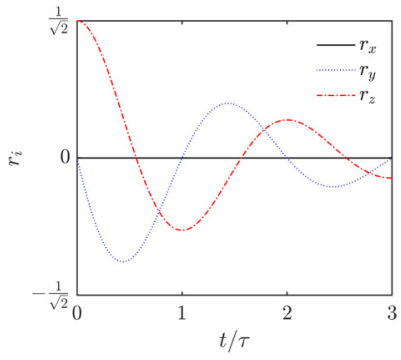

(d) Bloch vector components $r_{i}$
FIG. 1. Evolution speed for a PT-symmetric quantum system. The behavior of the evolution speed $v$ as a function of time is shown when the system is initialized in the spin- $z$ up state (a). The radial $v_{R}$ in panel (b) and tangential $v_{T}$ in panel (c) components are also shown. Results for the decay rates $\gamma=0.2,1,4$, corresponding to the unbroken, critical and broken PT-symmetry phases, are shown in each plot when $g=1$. The components of the Bloch vector in the unbroken phase are also pictured in panel (d). Time is measured in units of the period $\tau=\pi / \sqrt{g^{2}-\gamma^{2}}$ with $\gamma=0.2$.

and the four eigenvalues of the Liouville operator are thus given by $0,-2 \gamma$, and $-\gamma \pm \sqrt{\gamma^{2}-g^{2}}$. We see that in the region of unbroken PT symmetry where $g>\gamma$ the eigenvalues are either real or come in complex conjugate pairs; at the exceptional point $g=\gamma$ the PT symmetry gets broken; and in the symmetry-broken phase where $g<\gamma$ all the eigenvalues are real. We expect to observe different behaves of the system in each of these phases. Indeed, the solutions to (5) are given by $r_{x}(t)=e^{-2 \gamma t} r_{x}(0), \quad r_{y}(t)=e^{-\gamma t}[(\cos \omega t-$ $\left.(\gamma / \omega) \sin \omega t) r_{y}(0)-(g / \omega) \sin \omega t r_{z}(0)\right]$ and $r_{z}(t)=$ $e^{-\gamma t}\left[(g / \omega) \sin \omega t r_{y}(0)+(\cos \omega t+(\gamma / \omega) \sin \omega t) r_{z}(0)\right]$, with $\omega=\sqrt{g^{2}-\gamma^{2}}$. They are oscillatory in the unbroken phase $g>\gamma$, whereas in the broken phase $g<\gamma$ the oscillations associated with the unitary part are completely suppressed.

The speed and the corresponding radial component, as well as the tangential component $v_{T}^{2}(t)=v^{2}(t)-v_{R}^{2}(t)$, are obtained by inserting these expressions in Eqs. (9) and (12). The components of the speed are shown in Fig. 1 for a system prepared in the spin- $z$ up state $|\psi(0)\rangle=|\uparrow\rangle$. Because this is an eigenstate of $\hat{L}$, we have $v_{R}(t)=0$ at $t=0$. The behavior of the speed varies between the broken and unbroken PT phases. In the unbroken phase, the speed exhibits a decay superimposed with oscillations. Here, $v_{R}(t)$ oscillates periodically with the period $\tau=\pi / \sqrt{g^{2}-\gamma^{2}}$, where the minima correspond to the times at which the Bloch vector is aligned with the $z$ axis, i.e., when $\mathcal{D} \hat{\rho} \propto \hat{\rho}$. Moving into the broken phase, the speed decays rapidly at short times and the oscillation in $v_{T}(t)$ is completely damped out. However, in this phase

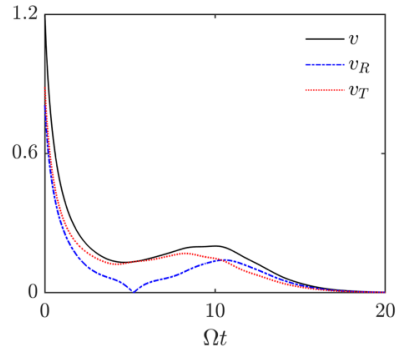

(a) Evolution speed

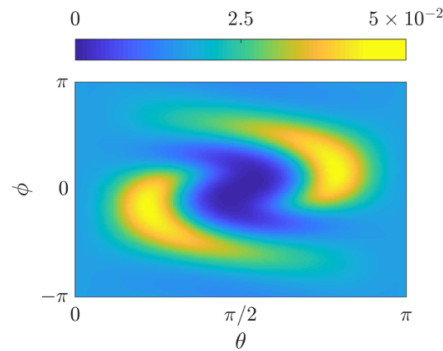

(c) $Q(\theta, \phi)$ at $\Omega t \approx 5$

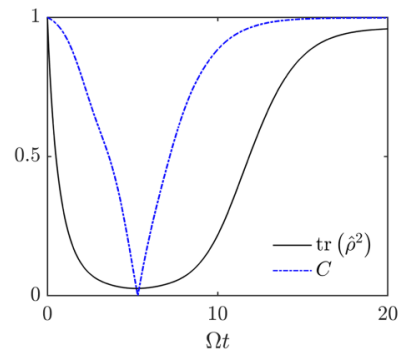

(b) Purity and phase coherence

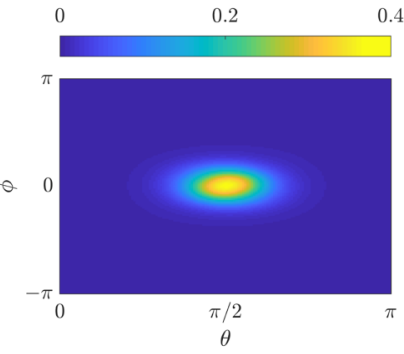

(d) $Q(\theta, \phi)$ at $\Omega t \approx 10$
FIG. 2. Evolution speed in an open BEC system. The evolution speed $v$, together with the radial $v_{R}$ and tangential $v_{T}$ components, is shown for an initial BEC state with the parameters $\theta=\pi / 2, \phi=$ $\pi$, and $N=50$ (a). The purity $\operatorname{tr}\left(\hat{\rho}^{2}\right)$ of the state and the phase coherence $C=2\left|\left\langle\hat{J}_{x}+i \hat{J}_{y}\right\rangle\right|\left(N^{2}-4\left\langle\hat{J}_{z}\right\rangle^{2}\right)^{-1 / 2}$ between the two sites are shown in (b). The Husimi function of the state at $\Omega t \approx 5$ and $\Omega t \approx 10$ is depicted in (c) and (d), respectively. In each plot time is measured in units of the inverse tunneling rate, $U N=0.8 \Omega$ and $\gamma N=0.8 \Omega$.

the velocity remains nonzero for a longer duration, with a small nonzero radial component remaining once the tangential component has virtually vanished.

In the previous examples, as well as a number of other similar examples we considered, the evolution speed is decreasing in time. Indeed, in Ref. [18] the Cauchy-Schwarz inequality is applied to obtain a bound on the speed of relative purity change, which shows that this speed is decreasing in time, and one might conjecture that the evolution speed of the state in general is also decreasing in time. However, this is not necessarily the case. We shall demonstrate this by means of a counterexample based on a driven dissipative Bose-Einstein condensate (BEC) in a two-site optical lattice, previously studied in the context of dissipative state preparation [27]. The unitary dynamics are generated by the Bose-Hubbard Hamiltonian $\hat{H}=-\Omega \hat{J}_{x}+U \hat{J}_{z}^{2}$, where $\Omega$ is the coupling strength between the two sites, $U \geqslant 0$ is the repulsive onsite interaction strength and the angular momentum operators $\hat{J}_{i}$ satisfy the $\mathfrak{s u}(2)$ commutation relations $\left[\hat{J}_{i}, \hat{J}_{j}\right]=i \varepsilon_{i j k} \hat{J}_{k}$. Coupling the bosons on the lattice to a reservoir leads to dissipation that can be described by the Lindblad operator $\hat{L}=\sqrt{\gamma}\left(\hat{J}_{z}-i \hat{J}_{y}\right)$. As the number operator $\hat{N}$ commutes with each $\hat{J}_{i}$, the particle number $N$ is conserved and we may thus restrict the analysis to the Hilbert subspace $\mathcal{H}^{N+1}$ of fixed particle number.

We worked out the evolution speed of an initial pure BEC state, represented by the $S U(2)$ coherent state $|\theta, \phi\rangle=\exp \left[i \theta\left(\hat{J}_{x} \sin \phi-\hat{J}_{y} \cos \phi\right)\right]|N, 0\rangle$, where the Fock 
state $|N, 0\rangle$ corresponds to all $N$ particles in the first lattice site. The result, plotted in Fig. 2, shows that the speed of evolution can increase. Initially, the Husimi (Fushimi) function $Q(\theta, \phi)=\langle\theta, \phi|\hat{\rho}| \theta, \phi\rangle$ of the state rapidly delocalizes in phase space (lower left panel) and the speed slows down. However, the distribution then spirals towards the origin (a sink in the semiclassical limit), and, depending on the parameter choice, at the beginning of this localisation the speed can temporarily increase. As the state tends towards the steady state the speed then decreases again to zero. The initial loss of phase coherence between the two sites (upper right panel) indicates destruction of the condensate. The point at which the phase coherence is completely lost is the point at which the radial component of the velocity goes to zero, and the point at which the state is most mixed.

In summary, we have derived a closed-form expression for the evolution speed $v(t)$ of the state, which shows that it consists of three terms corresponding to the unitary contribution, the Lindblad contribution, and the competition of the two. We have also worked out the radial component, connected to the purity change, and showed that this is given by the modified skew information for the Lindblad operators. We examined example systems that show that the speed of evolution is typically decreasing in time, but this need not be the case in general. Our results on the evolution speed open up a new challenge of maximising $v(t)$ over all Liouville operators $\mathcal{L}$ under suitable constraints, as well as solving the open-system quantum navigation problem.

Note added. Recently, we came across a closely related work [28], in which the Euclidean norm is used to investigate bounds on the evolution time for general open systems. Various merits in the use of the Euclidean norm are also discussed therein.

\section{ACKNOWLEDGMENTS}

B.L. acknowledges Engineering and Physical Sciences Research Council for support under the Doctoral Training Partnership (Grant No. EP/M507878/1). The authors thank E. M. Graefe for useful comments.
[1] L. Mandelstam and I. Tamm, The uncertainty relation between energy and time in nonrelativistic quantum mechanics, J. Phys. A (USSR) 9, 249 (1945).

[2] J. Uffink, The rate of evolution of a quantum state, Am. J. Phys. 61, 935 (1993).

[3] D. C. Brody and L. P. Hughston, Geometry of Quantum Statistical Inference, Phys. Rev. Lett. 77, 2851 (1996).

[4] J. Anandan and Y. Aharonov, Geometry of Quantum Evolution, Phys. Rev. Lett. 65, 1697 (1990).

[5] D. C. Brody, Information geometry of density matrices and state estimation, J. Phys. A 44, 252002 (2011).

[6] S. Luo, Wigner-Yanase Skew Information and Uncertainty Relations, Phys. Rev. Lett. 91, 180403 (2003).

[7] S. L. Braunstein and C. M. Caves, Statistical Distance and the Geometry of Quantum States, Phys. Rev. Lett. 72, 3439 (1994).

[8] D. Mondal and A. K. Pati, Quantum speed limit for mixed states using an experimentally realizable metric, Phys. Lett. A 380, 1395 (2016).

[9] S. Deffner and S. Campbell, Quantum speed limits: from Heisenberg's uncertainty principle to optimal quantum control, J. Phys. A 50, 453001 (2017).

[10] D. C. Brody, Geometry of the complex extension of Wigner's theorem, J. Phys. A 46, 395301 (2013).

[11] S. Alipour, M. Mehboudi, and A. T. Rezakhani, Quantum Metrology in Open Systems: Dissipative Cramér-Rao Bound, Phys. Rev. Lett. 112, 120405 (2014).

[12] K. Funo, N. Shiraishi, and K. Saito, Speed limit for open quantum systems, New J. Phys. 21, 013006 (2019).

[13] R. Uzdin and R. Kosloff, Speed limits in Liouville space for open quantum systems, Europhys. Lett. 115, 40003 (2016).

[14] S. Deffner and E. Lutz, Quantum Speed Limit for NonMarkovian Dynamics, Phys. Rev. Lett. 111, 010402 (2013).

[15] A. Uhlmann, The 'transition probability' in the state space of algebra, Rep. Math. Phys. 9, 273 (1976).

[16] R. Jozsa, Fidelity for mixed quantum states, J. Mod. Opt. 41, 2315 (1994).
[17] M. M. Taddei, B. M. Escher, L. Davidovich, and R. L. de Matos Filho, Quantum Speed Limit for Physical Processes, Phys. Rev. Lett. 110, 050402 (2013).

[18] A. del Campo, I. L. Egusquiza, M. B. Plenio, and S. F. Huelga, Quantum Speed Limit in Open System Dynamics, Phys. Rev. Lett. 110, 050403 (2013).

[19] D. C. Brody and E. M. Graefe, Mixed-State Evolution in the Presence of Gain and Loss, Phys. Rev. Lett. 109, 230405 (2012).

[20] D. C. Brody, G. W. Gibbons, and D. M. Meier, Time-optimal navigation through quantum wind, New J. Phys. 17, 033048 (2015).

[21] V. Mukherjee, A. Carlini, A. Mari, T. Caneva, S. Montangero, T. Calarco, R. Fazio, and V. Giovannetti, Speeding up and slowing down the relaxation of a qubit by optimal control, Phys. Rev. A 88, 062326 (2013)

[22] R. A. Bertlmann and P. Krammer, Bloch vectors for qudits, J. Phys. A 41, 235303 (2008).

[23] W. Pfeifer, The Lie Algebra su(N): An Introduction (Birkhäuser, Basel, 2003).

[24] G. Della Riccia and N. Wiener, Wave mechanics in classical phase space, Brownian motion, and quantum theory, J. Math. Phys. 7, 1372 (1966).

[25] B. Baumgartner, H. Narnhofer, and W. Thirring, Analysis of quantum semigroups with GKS-Lindblad generators: I. Simple generators, J. Phys. A 41, 065201 (2008).

[26] F. Campaioli, F. A. Pollock, F. C. Binder, and K. Modi, Tightening Quantum Speed Limits for Almost All States, Phys. Rev. Lett. 120, 060409 (2018).

[27] S. Diehl, A. Micheli, A. Kantian, B. Kraus, H. P. Büchler, and P. Zoller, Quantum states and phases in driven open quantum systems with cold atoms, Nat. Phys. 4, 878 (2008).

[28] F. Campaioli, F. A. Pollock, and K. Modi, Tight, robust, and feasible quantum speed limits for open dynamics, Quantum 3 , 168 (2019). 